\title{
A COMPOSITE SAMPLE METHOD FOR DETERMINATION OF GLOBAL TEXTURE WITHOUT SAMPLE SYMMETRY
}

\author{
X. ZHAO, L. ZUO, Z. LIANG and H. ZHAO \\ Department of Materials Science and Engineering Northeast University of \\ Technology, Shenyang, P.R. China
}

(Received March 28 1991)

\begin{abstract}
A new flexible composite sample method is described, which permits the global texture of a sheet material to be determined from one single composite sample. Its applicability is demonstrated by a practical example.
\end{abstract}

KEY WORDS Composite Sample Method, Global Texture, ODF

To predict some physical properties of a polycrystalline material, it requires the knowledge of its global texture. Several composite sample methods have been developed for this purpose (Meieran, 1962; Lopata and Kula, 1962; Leber, 1965; Elias and Heckler, 1967; Welch, 1980). Those methods were aimed to get complete pole figures merely by the Schulz back-reflection technique. Here a new composite sample method suggested in this paper is characterized by getting ODF

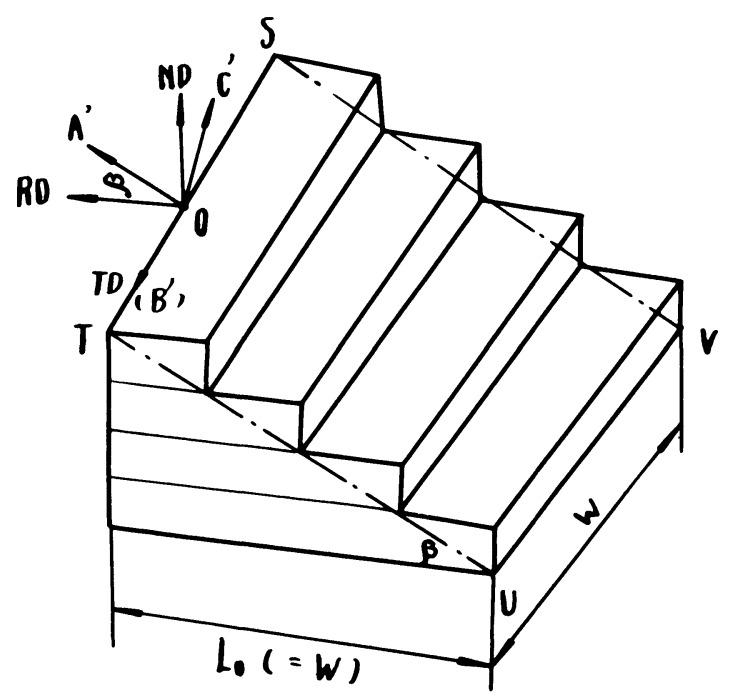

Figure 1 Preparation of the composite Sample 
from one sample directly, whether the texture of the test material possesses sample symmetry or not.

Preparation of a new composite sample is rather easy. Here $n$ rectangular pieces of equal width $W$ and different length $L\left(L=L_{0}, L_{0}(1-1 / n), L_{0}(1-\right.$ $\left.2 / n), \ldots, L_{0} / n\right)$ are cut from the tested sheet material with $L$ side parallel to the rolling direction (RD) of the sheet. These $n$ pieces are piled sequentially and glued together to form a wedge, as is schematically shown in Figure. 1. The oblique washboard-like surface of the wedge which is then ground and polished to become a smooth plane STUV, serves as the measured plane. Several incomplete pole figures can be measured, from which the series coefficients $W_{l p n}^{\prime}$ of the relative ODF, whose coordinate plane $O A^{\prime} B^{\prime}$ coincides with STUV, are derived. Finally, the desired series coefficients $W_{l m n}$ with RD, TD, and ND as the reference coordinate axes are calculated according to the relation (Roe, 1965;

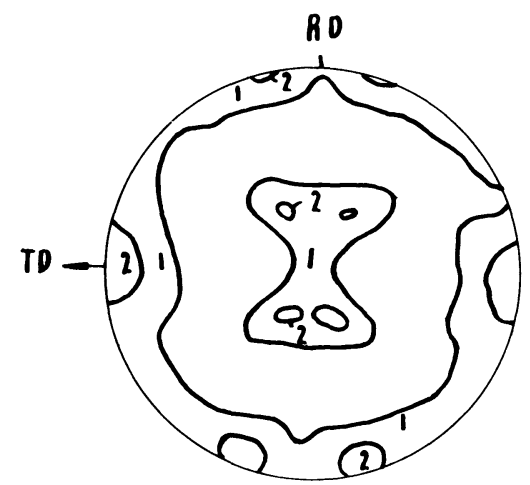

(a) $\beta=40^{\circ}$

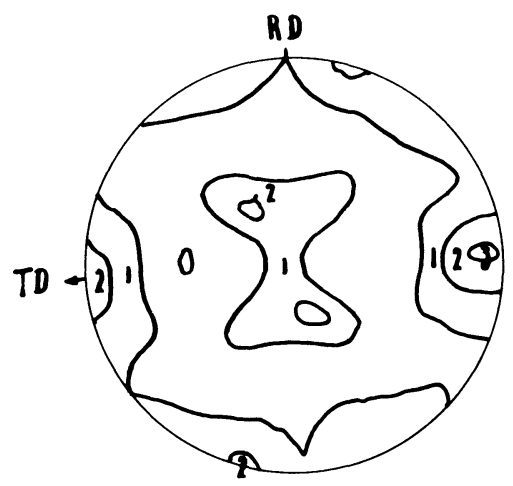

(C) $\beta=45^{\circ}$

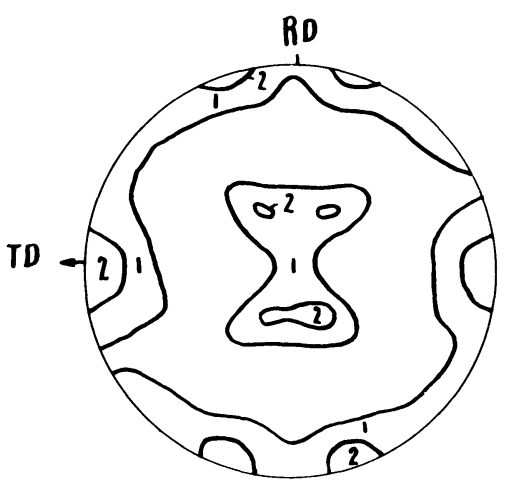

(b) $\beta=7.3^{\circ}$

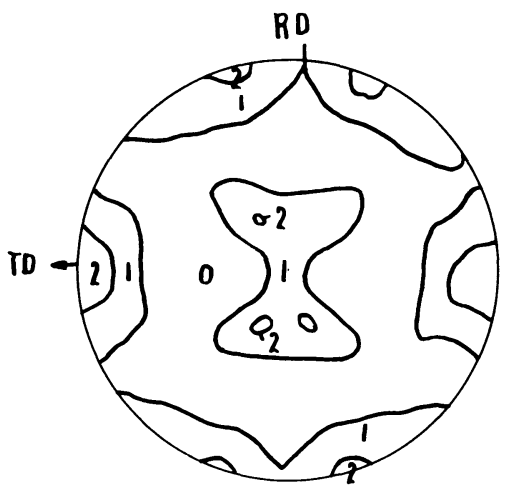

(d) $\beta=60^{\circ}$

Figure 2 Recalculated 111 pole figures 
also Bunge and Esling, 1985),

$$
W_{l m n}=\left(\frac{2}{2 l+1}\right)^{1 / 2} \sum_{p=-1}^{1} z_{l p m}(\cos \beta) W_{l p n}^{\prime}
$$

where $\beta$ is the measured value $\beta=\operatorname{tg}^{-1}\left(n d / L_{0}\right), d$ is the thickness of the test material. The value of $\beta$ is unrestricted except zero, so the angle error introduced from the preparation of the composite sample can be practically avoided.

As an example of examination of this method, the textures of four composite samples made from an $\alpha$-brass sheet cross-shear rolled in single direction have been determined. The thickness of the sheet is $0.3 \mathrm{~mm} ; L_{0}$ takes $25.7 \mathrm{~mm}$. These samples are piled up by using $6,11,86$ and 150 pieces of sheet respectively and their corresponding $\beta$ are $4^{\circ}, 7.3^{\circ}, 45^{\circ}$ and $60^{\circ}$. The determined ODFs of these samples coincide with each other rather well, each recalculated (111) pole figure being illustrated in Figure 2.

Hence, it may be concluded that the present method is suitable for practical use and its reliability is satisfactory.

\section{Acknowledgement}

This work is financially supported by the National Natural Science Foundation of China, Grant No. 5870028 .

\section{References}

1. Bunge, H. J. and Esling, C. (1985). Preferred Orientation in Deformed Metals and Rocks, Ed. Wenk H. R., Academic Press, Orlando, 114.

2. Elias, J. A. and Heckler, A. J. (1967). Trans. TMS-AIME, 239, 1237.

3. Leber, S. (1965). Rev. Sci. Instr., 36, 1747.

4. Lopata, S. L. and Kula, E. B. (1962). Trans. TMS-AIME, 224, 865.

5. Meieran, E. S. (1962). Rev. Sci. Inetr., 33, 319.

6. Roe, R. J. (1965). J. Appl. Phys., 36, 2024.

7. Welch, P. I. (1980). Texture Cryst. Sol., 4, 99. 